\title{
Serine and alanine racemase activities of VanT: a protein necessary for vancomycin resistance in Enterococcus gallinarum BM4174
}

\author{
Cesar A. Arias, $\uparrow$ Jan Weisner, Jonathan M. Blackburn \\ and Peter E. Reynolds
}

Department of

Biochemistry, University of

Cambridge, Tennis Court

Road, The Downing Site,

Cambridge CB2 1QW, UK
Author for correspondence: Cesar A. Arias. Tel: +57 1633 1512. Fax: +1 9174773388. e-mail:caa22@cable.net.co

Vancomycin resistance in Enterococcus gallinarum results from the production of UDP-MurNAc-pentapeptide[D-Ser]. VanT, a membrane-bound serine racemase, is one of three proteins essential for this resistance. To investigate the selectivity of racemization of L-Ser or L-Ala by VanT, a strain of Escherichia coli TKL-10 that requires D-Ala for growth at $42{ }^{\circ} \mathrm{C}$ was used as host for transformation experiments using plasmids containing the full-length vanT from Ent. gallinarum or the alanine racemase gene (alr) of Bacillus stearothermophilus: both plasmids were able to complement $E$. coli TKL-10 at $42{ }^{\circ} \mathrm{C}$. No alanine or serine racemase activities were detected in the host strain E. coli TKL-10 grown at 30, 34 or $37^{\circ} \mathrm{C}$. Serine and alanine racemase activities were found almost exclusively $(96 \%)$ in the membrane fraction of $E$. coli TKL10/pCA4(vanT): the alanine racemase activity of VanT was $14 \%$ of the serine racemase activity in both E. coli TKL-10/pCA4(vanT) and E. coli XL-1 Blue/pCA4(vanT). Alanine racemase activity was present mainly $(95 \%)$ in the cytoplasmic fraction of E. coli TKL-10/pJW40(alr), with a trace $(1.6 \%)$ of serine racemase activity. Additionally, DNA encoding the soluble domain of VanT was cloned and expressed in E. coli M15 as a His-tagged polypeptide and purified: this polypeptide also exhibited both serine and alanine racemase activities; the latter was approximately $18 \%$ of the serine racemase activity, similar to that of the full-length, membrane-bound enzyme. $\mathrm{N}$-terminal sequencing of the purified His-tagged polypeptide revealed a single amino acid sequence, indicating that the formation of heterodimers between subunits of His-tagged $C-V a n T$ and endogenous alanine racemases from $E$. coli was unlikely. The authors conclude that the membrane-bound serine racemase VanT also has alanine racemase activity but is able to racemize serine more efficiently than alanine, and that the cytoplasmic domain is responsible for the racemase activity.

Keywords: D-serine, vancomycin resistance, racemases, Enterococcus gallinarum

\section{INTRODUCTION}

Vancomycin is a glycopeptide antibiotic that inhibits synthesis of the bacterial cell wall by binding to the acylD-alanyl-D-alanine (D-Ala-D-Ala) moiety of peptidoglycan precursors (Reynolds, 1989). Vancomycin resistance in Enterococcus gallinarum results from the synthesis of precursors ending in $\mathrm{D}$-serine (D-Ser) (Reynolds

†Present address: Centro de Investigaciones, Universidad El Bosque, Transversal 9A no. 133-25, Santafé de Bogotá, Colombia. et al., 1994; Billot-Klein et al., 1994a): the antibiotic has a lower affinity for UDP-MurNAc-pentapeptide[Ser] (the main peptidoglycan precursor synthesized) than for UDP-MurNAc-pentapeptide[Ala] (Billot Klein et al., 1994b). Resistance requires the presence of three proteins (Arias et al., 2000): VanC-1 synthesizes the dipeptide D-Ala-D-Ser (Park et al., 1997) that is incorporated into peptidoglycan precursors; $\operatorname{VanXY}_{\mathrm{C}}$ hydrolyses the dipeptide D-Ala-D-Ala and removes DAla from peptidoglycan precursors ending in D-Ala, ensuring the removal of 'susceptible' precursors 
(Reynolds et al., 1999); and VanT converts L-serine (LSer) to D-Ser for peptidoglycan synthesis (Arias et al., 1999).

D-Ser is not a naturally occurring amino acid, although it is produced spontaneously in the mammalian stomach due to low $\mathrm{pH}$ values, and it has also been reported to be a growth inhibitor of Escherichia coli in minimal media (McFall \& Newman, 1996). Its presence has been reported in E. coli strains, where it serves as a readily available source of carbon (Gutnick et al., 1969): a specific deaminase system converts D-Ser to pyruvate (Dowhan \& Snell, 1970). Alanine racemases are pyridoxal-phosphate-dependent enzymes that catalyse the production of D-Ala from its enantiomer precursor LAla (Walsh, 1989). Two different alanine racemases (anabolic and catabolic) have been characterized in Salmonella typhimurium and E. coli (Wasserman et al., 1983; Wild et al., 1985) whereas in other bacteria investigated only one alanine racemase has been detected. The serine racemase activity of the anabolic enzyme from S. typhimurium involved in the synthesis of peptidoglycan was only $15 \%$ of the alanine racemase activity (Esaki \& Walsh, 1986). In multicellular organisms pyridoxal-phosphate-dependent serine racemases have been characterized in the silkworm Bombyx mori (Uo et al., 1998), where the concentration of $\mathrm{D}$-Ser is increased at particular stages of metamorphosis (Corrigan \& Srinivasan, 1966), and in mammalian brain (Wolosker et al., 1999a), where it functions as a neuromodulator at the 'glycine site' of the N-methyl-Daspartate receptor (Matsui et al., 1995). VanT is the only serine racemase identified in bacteria so far. Unlike other alanine and serine racemases described, VanT is a transmembrane protein: at least ten transmembrane helices are predicted to be present in the $\mathrm{N}$-terminal domain (Arias et al., 1999) and the C-terminal domain has structural homology with the alanine racemase from Bacillus stearothermophilus (Shaw et al., 1997), including conservation of the most important residues necessary for binding of the pyridoxal phosphate cofactor (Lys371) and for catalytic activity. Molecular modelling demonstrated that VanT could exist as a dimer (Arias et al., 1999). In this paper we demonstrate that VanT exhibits significant alanine racemase activity and confirm that the C-terminal domain is sufficient for racemase activity.

\section{METHODS}

Bacterial strains and growth conditions. E. coli TKL-10 has a strict requirement for $\mathrm{D}-\mathrm{Ala}$ to grow at $42{ }^{\circ} \mathrm{C}$ due to the presence of at least three mutations affecting alanine metabolism (Wijsman, 1972; Wasserman et al., 1983). This strain and E. coli XL-1 Blue (Bullock et al., 1987) were grown at $34^{\circ} \mathrm{C}$ in Luria-Bertani (LB) broth or agar and E. coli M15[pREP4] (Qiagen) was grown at $37^{\circ} \mathrm{C}$ in LB broth containing sorbitol $(0.5 \mathrm{M})$ and betaine $(2.5 \mathrm{mM})$. Ampicillin $\left(100 \mu \mathrm{g} \mathrm{ml}^{-1}\right)$ was added for plasmid-containing derivatives of E. coli TKL-10 and E. coli XL-1 Blue and both ampicillin $\left(100 \mu \mathrm{g} \mathrm{ml}^{-1}\right)$ and kanamycin $\left(25 \mu \mathrm{g} \mathrm{m} \mathrm{m}^{-1}\right)$ were added for plasmid-containing derivatives of E. coli M15[pREP4].

Functional complementation experiments were carried out in
E. coli TKL-10 and the same strain containing plasmid constructs expressing vanT (pCA4) and alr (pJW40). Bacteria were grown at $42{ }^{\circ} \mathrm{C}$ (non-permissive temperature) and at $30{ }^{\circ} \mathrm{C}$ simultaneously in LB agar and broth and incubated for at least $72 \mathrm{~h}$.

DNA manipulations and plasmid construction. Cloning, digestion with restriction endonucleases, isolation of plasmid DNA, transformation and ligations were carried out by standard methods (Sambrook et al., 1989). Plasmids pCA4 and pCA5 have been described previously (Arias et al., 1999). For construction of plasmid pJW40, the alr gene from $B$. stearothermophilus was amplified from total DNA with primer A (5'-GATCGATCGATCCATATGAACGACTTTCATCGCGATACG-3'), which includes a NdeI site (underlined), and primer B (5'-GATCGAGGATCCAAGCTTTTAGTGATGGTGATGGTGATGTGCACTGCTTTCCCCGCGGCC-3'), which includes a BamHI site (underlined), a HindIII site (bold) and the sequence encoding six histidines followed by a stop codon (italics). The PCR product was cloned as a NdeI-HindIII fragment into a derivative of pUC19 (Norrander et al., 1983) under the control of the trc promoter from pKK233-2 (Amann \& Brosius, 1985).

Preparation of membrane and cytoplasmic extracts. E. coli TKL-10, E. coli XL-1 Blue and derivatives were grown in LB medium $(25 \mathrm{ml})$ at $34{ }^{\circ} \mathrm{C}$ with aeration, with corresponding antibiotics if needed. When the $\mathrm{OD}_{600}$ had reached $0 \cdot 8,0 \cdot 2 \mathrm{mM}$ IPTG was added and incubation continued for $2 \cdot 5 \mathrm{~h}$. Bacteria were harvested, washed in $150 \mathrm{mM}$ Bistris propane buffer $(\mathrm{pH}$ $7 \cdot 5)$, resuspended in $1 \mathrm{ml}$ of the same buffer and sonicated. The broken cell preparation was centrifuged at $100000 \mathrm{~g}$ for $60 \mathrm{~min}$, the supernatant (cytoplasmic fraction) was collected and the pellet (membrane fraction) was washed in $150 \mathrm{mM}$ Bistris propane buffer $(\mathrm{pH} 7 \cdot 5)$ before final resuspension in $200 \mu \mathrm{l}$ of the same buffer.

Purification of the C-terminal domain of VanT. E. coli M15[pREP4] (Qiagen) containing plasmid pCA5 encoding the His-tagged C-terminal domain of $\operatorname{VanT}(\mathrm{C}-\mathrm{VanT})$ (Arias et al., 1999) was grown at $37^{\circ} \mathrm{C}$ in LB broth $(300 \mathrm{ml})$ containing sorbitol $(0.5 \mathrm{M})$ and betaine $(2.5 \mathrm{mM})$ to favour the synthesis of soluble protein (Blackwell \& Horgan, 1991), due to the fact that initial purification experiments indicated the presence of inclusion bodies when the gene was expressed after induction with IPTG. When the $\mathrm{OD}_{600}$ had reached 1.0, 0.05 mM IPTG was added and incubation continued for $40 \mathrm{~min}$. Bacteria were harvested at $4{ }^{\circ} \mathrm{C}$, washed in lysis buffer $(50 \mathrm{mM}$ Bistris propane $/ 300 \mathrm{mM} \mathrm{NaCl} / 10 \mathrm{mM}$ imidazole, pH 8.0) resuspended in $5 \mathrm{ml}$ of the same buffer and sonicated. The broken cell preparation was centrifuged at $40000 \mathrm{~g}$ for $20 \mathrm{~min}$ at $4{ }^{\circ} \mathrm{C}$ and the supernatant collected. All the following steps were carried out at $4{ }^{\circ} \mathrm{C}$. The supernatant was applied to a $2 \mathrm{ml}$ nickel-containing agarose column (Agarose Ni-NTA, Qiagen) equilibrated with the same buffer. The column was washed with at least $100 \mathrm{ml} 50 \mathrm{mM}$ Bistris propane/300 mM NaCl/ $30 \mathrm{mM}$ imidazole buffer $(\mathrm{pH} 8)$ and His-tagged C-VanT eluted with $50 \mathrm{mM}$ Bistris propane $/ 300 \mathrm{mM} \mathrm{NaCl} / 250 \mathrm{mM}$ imidazole buffer $(\mathrm{pH} 8 \cdot 0)$. Fractions of $0.5 \mathrm{ml}$ were collected and assayed for serine racemase activity (see below).

Electrophoresis. SDS-PAGE on a $12 \%$ polyacrylamide gel was carried out under denaturing conditions using the Laemmli buffer system (Laemmli, 1970) to determine the purity and $M_{\mathrm{r}}$ of C-VanT. For calibration, standard proteins in the range of $M_{\mathrm{r}}$ 9000-175000 (New England Biolabs) were used. Proteins were stained with $0.1 \%(\mathrm{w} / \mathrm{v})$ Coomassie blue in $50 \%(\mathrm{v} / \mathrm{v})$ methanol $/ 10 \%(\mathrm{v} / \mathrm{v})$ acetic acid for $30 \mathrm{~min}$ at $37^{\circ} \mathrm{C}$ and destained with $10 \%(\mathrm{v} / \mathrm{v})$ methanol $/ 10 \%(\mathrm{v} / \mathrm{v})$ acetic acid at room temperature overnight. 
$\mathbf{N}$-terminal sequencing. The possibility of heterodimer formation between subunits of C-VanT and endogenous alanine racemases of $E$. coli was investigated by $\mathrm{N}$-terminal sequencing of the major band corresponding to the His-tagged CVanT $\left(M_{r}\right.$ 43087) and a band with an $M_{r}$ similar to the anabolic or catabolic alanine racemases $M_{\mathrm{r}} 39128$ and 38819 respectively) of E. coli. Proteins present in the sample containing the purified His-tagged C-VanT were precipitated with $10 \%(\mathrm{v} / \mathrm{v})$ trichloroacetic acid (TCA), separated on a $12 \%$ SDS-PAGE gel and electrotransferred to a PVDF membrane. The membrane was stained with $0 \cdot 1 \%(\mathrm{w} / \mathrm{v})$ Coomassie blue in $50 \%(\mathrm{v} / \mathrm{v})$ methanol $/ 1 \%(\mathrm{v} / \mathrm{v})$ acetic acid, destained with $50 \%$ methanol, washed in distilled water and dried. The upper and lower portions of the His-tagged CVanT and of a band with slightly greater mobility $\left(M_{\mathrm{r}}\right.$ approx. 39000) were cut out and subjected to micro-sequencing by sequential Edman degradation on a 477A sequencer in tandem with a model 120A analyser (Applied Biosystems).

Enzyme assays. Serine and alanine racemase activities of suitable dilutions of cytoplasmic and membrane fractions and of purified C-VanT were determined in a final volume of $30 \mu \mathrm{l}$. The assay mixture contained $100 \mathrm{mM}$ Bistris propane $\mathrm{pH} 7 \cdot 5$, $10 \mathrm{mM}$ L-serine or L-alanine and $10 \mu \mathrm{l}$ of the diluted fraction as enzyme preparation. Mixtures were incubated at $37^{\circ} \mathrm{C}$ for $40 \mathrm{~min}$ for the cytoplasmic and membrane preparations, and for $30 \mathrm{~min}$ at $37^{\circ} \mathrm{C}$ and $42{ }^{\circ} \mathrm{C}$ for purified C-VanT. D-Amino acids produced by racemase activity were assayed using a Damino acid oxidase assay (Messer \& Reynolds, 1992), with Dserine or D-alanine as standards. The lower reliable limit of detection of $\mathrm{D}$-Ser was $4 \mathrm{nmol} \mathrm{min}^{-1}(\mathrm{mg} \text { membrane protein })^{-1}$ and for D-Ala, $2 \mathrm{nmol} \mathrm{min}^{-1}$ (mg cytoplasmic protein $)^{-1}$. Protein concentration was determined according to the method of Bradford (1976), with bovine serum albumin as standard.

\section{RESULTS}

\section{Functional complementation of $E$. coli TKL-10 at $42{ }^{\circ} \mathrm{C}$}

E. coli TKL-10 is unable to grow at $42{ }^{\circ} \mathrm{C}$ in the absence of D-Ala (Wijsman, 1972). The mutations in the alanine racemases prevent the utilization of $\mathrm{L}-\mathrm{Ala}$ as the source of D-Ala for peptidoglycan synthesis at the non-permissive temperature $\left(42{ }^{\circ} \mathrm{C}\right.$ ) (Wijsman, 1972; Wassermann et al., 1983). The strain did not grow at $42{ }^{\circ} \mathrm{C}$ in the absence of an external supply of $\mathrm{D}$-Ala when an enrichment medium (LB) was used (Table 1). Growth at $42{ }^{\circ} \mathrm{C}$ without a supplement of $\mathrm{D}$-Ala was restored when E. coli TKL-10 was transformed with plasmids pCA4 or pJW40, which express vanT and alr respectively (Table 1 ), indicating that a basal level of expression (i.e. without induction) provided enough D-Ala for cell wall synthesis; this result also indicated that VanT was likely to possess alanine racemase activity.

\section{Alanine racemase activities of membrane and cytoplasmic fractions}

The majority $(95 \%)$ of the alanine racemase activity of E. coli TKL-10/pJW40(alr) was present in the cytoplasmic fraction after induction with IPTG, whereas it was not detectable in either cytoplasmic or membrane fractions of E. coli TKL-10 (Table 1). The amount of alanine racemase activity in the construct carrying the alr gene was 150-fold greater than that present in E. coli XL-1 Blue/pUC18, in which the enzyme, present exclusively in the cytoplasm (Table 1) is chromosomally encoded. A similar value for the activity of the cytoplasmic alanine racemase of E. coli XL-1 Blue/ pCA4(vanT) was obtained as with the strain lacking the van $T$ gene but, additionally, $24 \%$ of the total alanine racemase activity was found in the membrane fraction, presumably catalysed by VanT (Table 1). Confirmation that $\mathrm{VanT}$ had significant alanine racemase activity was obtained with E. coli TKL-10/pCA4(vanT) : almost all $(95 \%)$ the alanine racemase activity of this construct was present in the membrane fraction after induction with IPTG, a value identical to that for the distribution of serine racemase activity (Table 2 ).

\section{Serine racemase activities of membrane and cytoplasmic fractions}

As expected, no serine racemase activity was detected in E. coli TKL-10 or E. coli XL-1 Blue/pUC18. In the E. coli constructs containing the full-length van $T$ gene, the serine racemase activity was exclusively ( $97 \%$ ) present

Table 1. Alanine racemase activities of membrane and cytoplasmic fractions of $E$. coli TKL-10 and $E$. coli XL-1 Blue and derivatives, and growth at $42{ }^{\circ} \mathrm{C}$ (non-permissive temperature for $E$. coli TKL-10)

Values represent total activities in each fraction and are the mean and standard deviation of three independent experiments with assays in duplicate. Bacteria were grown at $34^{\circ} \mathrm{C}$. ND, Not detectable.

\begin{tabular}{|c|c|c|c|}
\hline \multirow[t]{2}{*}{ E. coli strain } & \multicolumn{2}{|c|}{ Alanine racemase activity $\left(\mathrm{nmol} \mathrm{min}^{-1}\right)$ in: } & \multirow{2}{*}{$\begin{array}{l}\text { Growth } \\
\text { at } 42{ }^{\circ} \mathrm{C}\end{array}$} \\
\hline & Membrane fraction & Cytoplasmic fraction & \\
\hline TKL-10 & ND & ND & - \\
\hline TKL-10/pJW40(alr) & $7900 \pm 990$ & $151600 \pm 9870$ & + \\
\hline TKL-10/pCA4 $(\operatorname{van} T)$ & $740 \pm 125$ & $36 \pm 6$ & + \\
\hline XL-1 Blue/pCA4 (vanT) & $336 \pm 69$ & $1020 \pm 92$ & + \\
\hline XL-1 Blue/pUC18 & $10 \pm 1$ & $990 \pm 58$ & + \\
\hline
\end{tabular}


Table 2. Distribution of serine and alanine racemase activities between membrane (M) and cytoplasmic (C) fractions of E. coli TKL-10 and E. coli XL-1 Blue and derivatives, and activity of one enzyme as a percentage of the other activity

\begin{tabular}{|c|c|c|c|c|c|c|c|c|}
\hline \multirow[t]{2}{*}{ E. coli strain } & \multicolumn{2}{|c|}{$\begin{array}{c}\text { Serine } \\
\text { racemase }(\%)^{*}\end{array}$} & \multicolumn{2}{|c|}{$\begin{array}{c}\text { Alanine } \\
\text { racemase }(\%) *\end{array}$} & \multicolumn{2}{|c|}{$\begin{array}{c}\text { Ala racemase } \\
\text { activity as \% } \\
\text { of Ser } \\
\text { racemase } \\
\text { activity }\end{array}$} & \multicolumn{2}{|c|}{$\begin{array}{c}\text { Ser racemase } \\
\text { activity as \% } \\
\text { of Ala } \\
\text { racemase } \\
\text { activity }\end{array}$} \\
\hline & M & $\mathrm{C}$ & M & $\mathrm{C}$ & M & $\mathrm{C}$ & M & $\mathrm{C}$ \\
\hline TKL-10 & ND & ND & ND & ND & & & & \\
\hline TKL-10/pJW40(alr) & 4 & 96 & 5 & 95 & - & - & $1 \cdot 5$ & $1 \cdot 6$ \\
\hline TKL-10/pCA4(vanT) & 97 & 3 & 95 & 5 & $13 \cdot 7$ & - & - & - \\
\hline XL-1 Blue/pCA4 (vanT) & 100 & 0 & 24 & 76 & $13 \cdot 7$ & - & - & - \\
\hline XL-1 Blue/pUC18 & - & - & 1 & 99 & - & - & - & 0 \\
\hline
\end{tabular}

* Percentage values were calculated from the means of total activities in each fraction. ND, Not detectable.

Table 3. Serine racemase activities of membrane and cytoplasmic fractions of $E$. coli TKL-10 and E. coli XL-1 Blue and derivatives

Values represent total activities in each fraction and are the mean and standard deviation of three independent experiments with assays in duplicate. Bacteria were grown at $34^{\circ} \mathrm{C}$. ND, Not detectable.

\begin{tabular}{|c|c|c|}
\hline \multirow[t]{2}{*}{ E. coli strain } & \multicolumn{2}{|c|}{$\begin{array}{l}\text { Serine racemase activity } \\
\quad\left(\mathrm{nmol} \mathrm{min}^{-1}\right) \text { in: }\end{array}$} \\
\hline & $\begin{array}{c}\text { Membrane } \\
\text { fraction }\end{array}$ & $\begin{array}{c}\text { Cytoplasmic } \\
\text { fraction }\end{array}$ \\
\hline TKL-10 & ND & ND \\
\hline TKL-10/pJW40(alr) & $117 \pm 10$ & $2510 \pm 32$ \\
\hline TKL-10/pCA4(vanT) & $5380 \pm 805$ & $142 \pm 27$ \\
\hline XL-1 Blue/pCA4 $(\operatorname{van} T)$ & $2450 \pm 300$ & $\mathrm{ND}$ \\
\hline XL-1 Blue/pUC18 & $3 \pm 1$ & $\mathrm{ND}$ \\
\hline
\end{tabular}

in the membrane fraction (Table 2). The activity of the plasmid-encoded serine racemase in both strains was substantial (two- to fivefold greater than that of the chromosomally encoded alanine racemase of E. coli XL1 Blue) but less than $4 \%$ of the cytoplasmic alanine racemase of E. coli TKL-10/pJW40(alr), which was also plasmid-encoded (Table 3). The limitation in amount of VanT may have resulted from the membrane localization of the enzyme: space considerations would most likely limit the production of large amounts. The membrane-bound alanine racemase activity of both $E$. coli TKL-10 and E. coli XL-1 Blue carrying vanT on plasmids was $14 \%$ of the serine racemase activity (Table 2 ). The high value for alanine racemase activity in the cytoplasm of E. coli TKL-10/pJW40(alr) enabled a reliable determination to be made of the serine racemase
Table 4. Serine and alanine racemase activities of the purified C-terminus of VanT

Values represent the mean and standard deviation of three independent preparations of purified His-tagged C-terminal domain of VanT. Percentage values are in relation to serine racemase activity at $37^{\circ} \mathrm{C}$.

\begin{tabular}{|lcc|}
\hline $\begin{array}{l}\text { Temp. } \\
\left({ }^{\circ} \mathrm{C}\right)\end{array}$ & $\begin{array}{c}\text { Serine racemase }(\mathbf{n m o l} \\
\left.\mathbf{m g}^{-1} \mathbf{m i n}^{-1}\right)[\%]\end{array}$ & $\begin{array}{c}\text { Alanine racemase }(\mathbf{n m o l} \\
\left.\mathbf{m g}^{-1} \mathbf{m i n}^{-1}\right)[\%]\end{array}$ \\
\hline 37 & $9877 \pm 688[100]$ & $1820 \pm 613[18 \pm 6 \cdot 2]$ \\
42 & $6934 \pm 812[70 \pm 8 \cdot 2]$ & $742 \pm 65[7 \cdot 5 \pm 0 \cdot 6]$ \\
\hline
\end{tabular}

activity (Table 3) attributable to the alanine racemase of B. stearothermophilus, namely $1.6 \%$ (Table 2 ), indicating that this racemase has a much stricter specificity than VanT. The alanine racemases of E. coli TKL10/pJW40(alr) and XL-1 Blue/pUC18 and the serine and alanine racemase activities of VanT were all inhibited by D-cycloserine: $50 \%$ inhibition occurred at between 1.4 and $2 \mathrm{mM}$ cycloserine in the presence of $10 \mathrm{mM}$ substrate.

\section{Alanine and serine racemase activities of purified C-VanT}

The alanine racemase activity of the purified His-tagged C-terminal domain of VanT (Fig. 1) was approximately $18 \%$ of the serine racemase activity (Table 4 ). Simultaneous assays of serine and alanine racemase activities performed at $37^{\circ} \mathrm{C}$ and $42^{\circ} \mathrm{C}$ indicated a loss of approximately $30 \%$ and $60 \%$ of the serine and alanine racemase activities, respectively, at $42^{\circ} \mathrm{C}$, in the $40 \mathrm{~min}$ assay (Table 4). The activities were stable at $4{ }^{\circ} \mathrm{C}$ but were lost on storage at $-20^{\circ} \mathrm{C}$. The findings indicate that the $\mathrm{C}$-terminal domain of VanT lacking the putative 


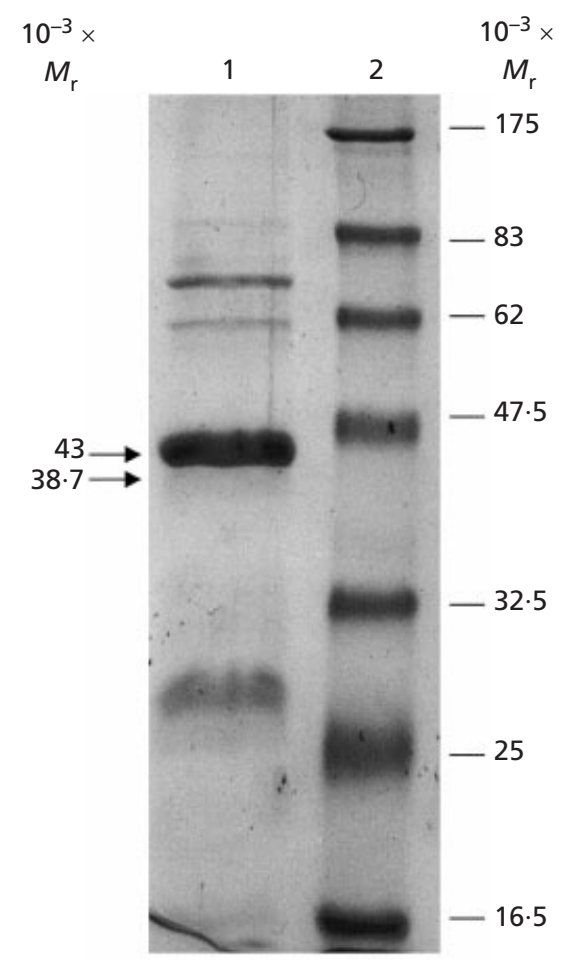

Fig. 1. SDS-PAGE of purified His-tagged C-terminal domain of VanT (His-tagged C-VanT). Lanes: 1, sample containing Histagged C-VanT $\left(M_{\mathrm{r}} 43000\right) ; 2$, standard proteins. The upper and lower regions of the band corresponding to His-tagged C-VanT were $\mathrm{N}$-terminally sequenced. A band of $M_{\mathrm{r}}$ approximately 39000 immediately below the band corresponding to C-VanT was also sequenced: the sequence matched the GldH (glycerol dehydrogenase) protein from E. coli (Truniger \& Boos, 1994).

10 transmembrane segments is sufficient for serine racemase activity.

\section{Purification of C-VanT and N-terminal sequencing}

Apart from the expected $M_{\mathrm{r}} 43000$ band corresponding to the His-tagged C-VanT, some contaminant proteins were present after purification: bands of $M_{\mathrm{r}} \geqslant 62000$ and $<32500$ were observed on SDS-PAGE in addition to the main $M_{r} 43000$ band (Fig. 1), although these were not considered likely to be involved in serine or alanine racemase activity. We also explored the possibility of heterodimer formation between the endogenous alanine racemase polypeptide and His-tagged C-VanT which could account for alanine racemase activity of the purified His-tagged protein. The calculated $M_{\mathrm{r}}$ of the purified His-tagged VanT is 43087 . The predicted $M_{\mathrm{r}}$ values of the endogenous anabolic and catabolic alanine racemases of E. coli are 39128 and 38819, respectively (Lobocka et al., 1994; Blattner et al., 1993). N-terminal sequencing was performed on both the upper and lower regions of the band corresponding to the His-tagged Cterminal domain of VanT and also on a band of slightly greater mobility (Fig. 1) (best observed when the purified protein was concentrated by TCA precipitation and separated under denaturing conditions on a SDS-PAGE gel). The sequence corresponding to the His-tagged CVanT was obtained from both upper and lower regions of the $M_{\mathrm{r}} 43000$ band (Fig. 1), and no evidence of a sequence corresponding to alanine racemases of E. coli was found. The sequence obtained from the band with $M_{\mathrm{r}}$ approximately 39000 (Fig. 1) yielded the first 15 amino acids of a protein that corresponded to the glycerol dehydrogenase $(\mathrm{GldH})$ from E. coli $\left(M_{\mathrm{r}} 38687\right)$ (Truniger \& Boos, 1994). It was concluded that the formation of heterodimers between subunits of alanine and serine racemase that could account for alanine racemase activity in the purified protein was unlikely.

\section{DISCUSSION}

VanT is an unique membrane-bound serine racemase that catalyses the synthesis of D-Ser in vancomycinresistant Ent. gallinarum (Arias et al., 1999). The synthesis of UDP-MurNAc-pentapeptide[D-Ser] and hydrolysis of 'susceptible' D-Ala-ending precursors is an absolute requirement for vancomycin resistance (Arias et al., 2000). Serine racemases have been described in eukaryotic cells, providing D-Ser for important physiological processes. In the silk worm Bombyx mori D-Ser may play an important role in development and metamorphosis (Corrigan \& Srinivasan, 1966). In mammalian brains D-Ser functions as an important neuromodulator (Wolosker et al., 1999a). Neither of these two enzymes appears to have a membrane association (Wolosker et al., 1999a; Uo et al., 1998). In E. coli D-Ser can act as a sole source of carbon and nitrogen (McFall \& Newman, 1996). However, D-Ser seems to be toxic for E. coli, inhibiting enzymes for panthothenate and Lserine synthesis (Cosloy \& McFall, 1973). Moreover, E. coli has a specific deaminase enzyme system for the catabolism of D-Ser (Dowhan \& Snell, 1970). It has been proposed that the natural source of D-Ser in E. coli is the non-enzymic racemization of L-Ser (Friedman, 1991). It is clear that vancomycin-resistant Ent. gallinarum has developed a specific enzyme to provide $\mathrm{D}$-Ser for peptidoglycan synthesis. The C-terminal domain of VanT has substantial sequence identity with alanine racemases of different organisms (Arias et al., 1999), especially with that of B. stearothermophilus, whose crystal structure has been solved (Shaw et al., 1997). Biochemical evidence presented here indicates that the C-terminal domain of VanT also catalyses the racemization of L-Ala. Alanine racemase activity was evident in vivo, when a plasmid expressing vanT was able to complement E. coli TKL-10 at the non-permissive temperature $\left(42{ }^{\circ} \mathrm{C}\right)$. Interestingly, we did not detect any alanine racemase activity in E. coli TKL-10 even at the permissive temperature $\left(30^{\circ} \mathrm{C}\right)$, a finding which is in agreement with the initial description of this strain by Wijsman (1972). Although the absence of enzyme activity at the permissive temperature has also been described in other temperature-sensitive mutants (Eidlic \& Neidhardt, 1965), it is likely that the sensitivity of the alanine racemase assay used here was insufficient to detect a basal, low activity of the enzyme, as has been 


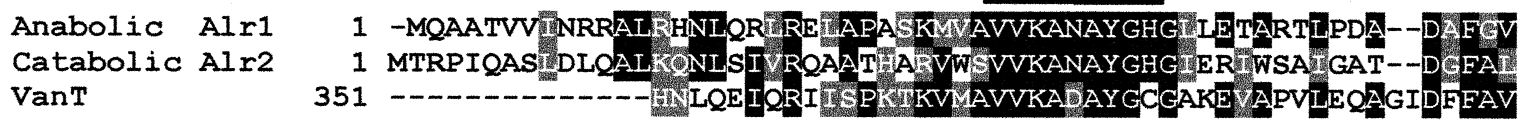

Anabolic Alr1 Catabolic Alr2 VanT

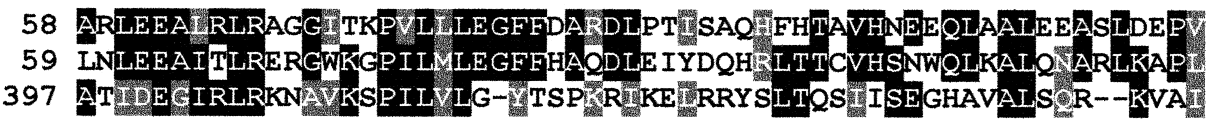

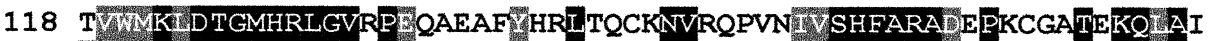 119 DIYLKVNSGMNLGFOPDRLTWWOLRAMANVGE T-LMSHFAEAEHPDG--ISGAMAR 454 DCHLAIDTGMHRLGVTPTIDSIISIFDLPFLTTSG Y SHDGSADRLNPDSMIRTEROIAC}

Anabolic Alr1 Catabolic Alr2 $\operatorname{Van} T$

\author{
Anabolic Alr1 \\ Catabolic Alr2 \\ $\operatorname{VanT}$
}

\begin{abstract}
Anabolic Alr1 Catabolic Alr2 $\operatorname{VanT}$
\end{abstract}

\section{FNTFCEGKPGQ-----RS MAASEGILLWPQSHFDWVRPGI I LYGVSPLEDRSTGADFGC

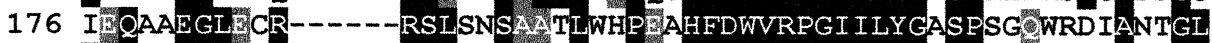

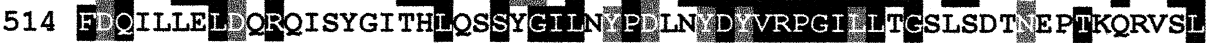

\section{QPVMSLMSSLI ARE HKAGEPVGYGGTVVSERDTRLGVVAMGYGDGYPRAAPSGTPVLVN 230 RPVMTLSSE I I VVTTIKAGERVGYGGR TARDEQR IGIVAAGY ADGY PRHAP IGTPVLVD

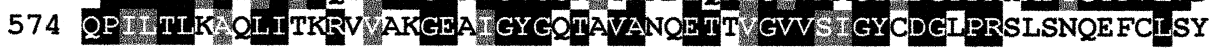
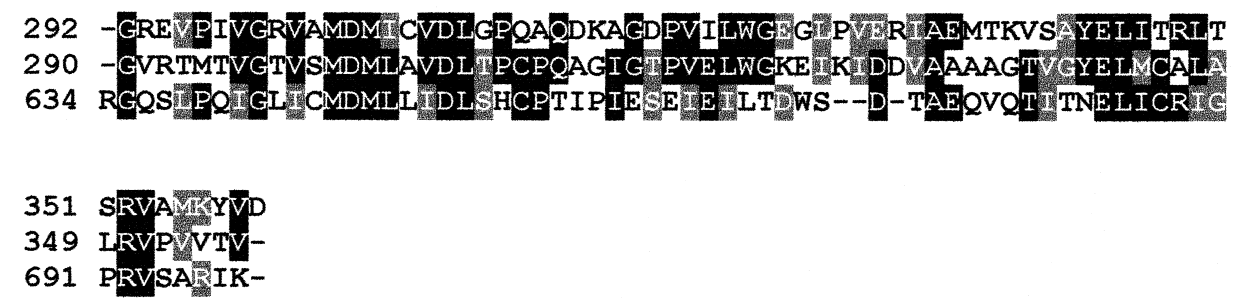

\section{PRVSAIK-}

Anabolic Alr1 Catabolic Alr2 VanT

Fig. 2. Alignment (CLUSTAL $W$; Thompson et al., 1994) of the C-terminal domain of VanT (GenBank accession no. AF 077816) with the anabolic (Alr1) and catabolic (Alr2) alanine racemases from E. coli (Wild et al., 1985). Black boxes indicate amino acid identity. Shaded boxes indicate similar amino acids. The putative pyridoxal phosphate attachment site of VanT, Alr 1 and Alr 2 is shown above the alignment. The predicted $\mathrm{N}$-terminal $\beta / \alpha$, and the C-terminal $\beta$ subdomains of VanT (Arias et al., 1999) include amino acids 351-558 and 575-698 respectively.

shown before in other strains of E. coli when the level of alanine racemase is below $4 \%$ of the normal activity (Wassermann et al., 1983). The appearance of such activity in the membrane fraction when the strain was transformed with pCA4 further supports the finding that full-length VanT also catalyses racemization of $\mathrm{L}^{-}$ Ala in vivo.

The alanine racemase activity of the purified His-tagged C-VanT was approximately $18 \%$ relative to its serine racemase activity (Table 4), similar to that of the membrane-bound enzyme (Table 2). Alanine racemase activities of the serine racemases of brain and silkworms are considerably less $(1.5 \%$ and $6 \%$ of the serine racemase activity respectively) (Wolosker et al., 1999a; Uo et al., 1998), indicating that the cytoplasmic domain of VanT is less selective than those of the eukaryotic enzymes. Recently, Wolosker et al. (1999b) reported the cloning and sequencing of the pyridoxal-phosphatedependent serine racemase from rat brain. Although the protein sequence does not exhibit significant homology with VanT it is likely that the mechanism of racemization of L-Ser is conserved in both proteins.

As VanT is likely to exist as a dimer (Arias et al., 1999) we explored the possibility of heterodimer formation between polypeptides of alanine racemase from E. coli and VanT. Cross-species heterodimers have been reported in different enzymes (Osterman et al., 1994; Sun et al., 1992; Greene et al., 1993). Active cross-species heterodimers are usually not formed if the subunits share less than $80 \%$ identity in the dimer interface (Osterman et al., 1994). An alignment between VanT and the alanine racemases of E. coli (Fig. 2) revealed that the overall sequence identity is only $33 \%$. Based on the crystal structure of Alr from B. stearothermophilus (Shaw et al., 1997) we previously predicted that the residues from the other subunit of VanT involved in binding or catalysis could be Tyr597, Asp647 and Met648 (replaced by Gln314 in Alr) (Arias et al., 1999), which are also conserved in the alanine racemases from E. coli (Fig. 2). However, it is unlikely that the sequence 
identity between the subunits of these proteins at the dimer interface is as high as $80 \%$. Also we did not find evidence of heterodimer formation based on $\mathrm{N}$-terminal sequencing of different parts of the band of the purified His-tagged C-VanT from the SDS-PAGE gel or from sequencing of a protein with a similar $M_{\mathrm{r}}$ to that of alanine racemase that was eluted with the purified Histagged C-VanT from the nickel column (Fig. 1). The sequence obtained did not correspond to alanine racemases (instead it matched a glycerol dehydrogenase). One possible explanation for the lack of detection of subunits of alanine racemases is that being in a small proportion compared to the His-tagged C-VanT, they undergo blocking at the $\mathrm{N}$-terminus and therefore become unavailable for Edman degradation. These results indicate that formation in vivo of heterodimers between subunits of alanine racemases of E. coli and the C-terminal domain of VanT is unlikely but if such heterodimers are present their proportion is likely to be very small compared to the formation of homodimers of $\mathrm{C}-\mathrm{V} a n T$. Another possibility is that the topological organization of the transmembrane and cytoplasmic domains of VanT may play a role in amino acid selectivity for racemization. Investigation of this interaction is currently in progress.

In summary, VanT is a membrane-bound serine racemase that also exhibits alanine racemase activity. The C-terminal domain of VanT is sufficient for catalysis and also has both activities.

\section{ACKNOWLEDGEMENTS}

C.A.A. is funded by COLCIENCIAS (Instituto Colombiano para el Desarrollo de la Ciencia y Tecnología 'Francisco José de Caldas') and the Overseas Research Scheme Award from the Committee of Vice-Chancellors and Principals of Universities in the United Kingdom. J.W. is funded by the European Commission via a Marie-Curie Fellowship. We thank M. Arthur and P. Courvalin for helpful discussions, and M. Weldon and C. Hill, Cambridge Center for Molecular Recognition, for N-terminal sequencing and synthesis of oligonucleotides respectively. We are grateful to the Cambridge Overseas Trust and the Lundgren Fund for personal financial assistance to C.A.A.

\section{REFERENCES}

Amann, E. \& Brosius, J. (1985). 'ATG Vectors' for regulated highlevel expression of cloned genes in Escherichia coli. Gene 40, 183-190.

Arias, C. A., Martín-Martinez, M., Blundell, T. L., Arthur, M., Courvalin, P. \& Reynolds, P. E. (1999). Characterization and modelling of VanT, a novel, membrane-bound, serine racemase from vancomycin-resistant Enterococcus gallinarum BM4174. Mol Microbiol 31, 1653-1664.

Arias, C. A., Courvalin, P. \& Reynolds, P. E. (2000). The van C gene cluster of Enterococcus gallinarum BM4174. Antimicrob Agents Chemother 44, 1660-1666.

Billot-Klein, D., Gutmann, L., Sable, S., Guitet, E. \& van Heijenoort, J. (1994a). Modification of peptidoglycan precursors is a common feature of the low-level vancomycin-resistant VANB-type Enterococcus D366 and of the naturally glycopeptide-resistant species Lactobacillus casei, Pediococcus pentosaceus, Leuconostoc mesenteroides and Enterococcus gallinarum. J Bacteriol 176, 2398-2405.

Billot-Klein, D., Blanot, D., Gutmann, L. \& van Heijenoort, J. (1994b). Association constants for the binding of vancomycin and teicoplanin to $\mathrm{N}$-acetyl-D-alanyl-D-alanine and $\mathrm{N}$-acetyl-Dalanyl-D-serine. Biochem J 304, 1021-1022.

Blackwell, J. R. \& Horgan, R. (1991). A novel strategy for production of a highly expressed recombinant protein in an active form. FEBS Lett 295, 10-12.

Blattner, F. R., Burland, V., Plunkett, G., Sofia, H. J. \& Daniels, D. L. (1993). Analysis of the Escherichia coli genome. 4. DNA sequence of the region from $89 \cdot 2$ to $92 \cdot 8$ minutes. Nucleic Acids Res 21, 5408-5417.

Bradford, M. M. (1976). A rapid and sensitive method for rapid quantitation of microgram quantities of protein utilising the principle of protein-dye binding. Anal Biochem 72, 248-254.

Bullock, W. O., Fernandez, J. M. \& Short, J. M. (1987). XL-1 Blue: a high-efficiency plasmid transforming recA Escherichia coli strain with beta-galactosidase selection. Biotechniques 5, 376 .

Corrigan, J. J. \& Srinivasan, N. G. (1966). The occurrence of certain D-amino acids in insects. Biochemistry 4, 1185-1190.

Cosloy, S. D. \& McFall, E. (1973). Metabolism of D-serine in Escherichia coli K-12: mechanism of growth inhibition. J Bacteriol 114, 685-694.

Dowhan, W., Jr \& Snell, E. E. (1970). D-Serine dehydratase from Escherichia coli. II. Analytical studies and subunit structure. $J$ Biol Chem 245, 4618-4628.

Eidlic, L. \& Neidhart, F. C. (1965). Protein and nucleic acid synthesis in two mutants of Escherichia coli with temperature-sensitive aminoacyl ribonucleic acid synthetases. J Bacteriol 89, 706-711.

Esaki, N. \& Walsh, C. T. (1986). Biosynthetic alanine racemase of Salmonella typhimurium: purification and characterization of the enzyme encoded by the alr gene. Biochemistry 25, 3261-3267.

Friedman, M. (1991). Formation, nutritional value, and safety of amino acids. In Nutritional and Toxicological Consequences of Food Processing, pp. 447-481. New York: Plenum.

Greene, P. J., Maley, F., Pedersen-Lane, J. \& Santi, D. V. (1993). Catalytically active cross-species heterodimers of thymidylate synthase. Biochemistry 32, 10283-10288.

Gutnick, D., Calvo, J. M., Klopotowski, T. \& Ames, B. N. (1969). Compounds which serve as the sole source of carbon or nitrogen for Salmonella typhimurium LT-2. J Bacteriol 100, 215-219.

Laemmli, U. K. (1970). Cleavage of structural proteins during the assembly of the head of bacteriophage T4. Nature 227, 680-685.

Lobocka, M., Hennig, J., Wild, J. \& Klopotowski, T. (1994). Organization and expression of the Escherichia coli K-12 dad operon encoding the smaller subunit of D-amino acid dehydrogenase and the catabolic alanine racemase. J Bacteriol 176, 1500-1510.

McFall, E. \& Newman E. B. (1996). Amino acids as carbon sources. In Escherichia coli and Salmonella: Cellular and Molecular Biology, pp. 358-379. Edited by F. C. Neidhart and others. Washington, DC: American Society for Microbiology.

Messer, J. \& Reynolds, P. E. (1992). Modified peptidoglycan precursors produced by glycopeptide-resistant enterococci. FEMS Microbiol Lett 94, 195-200.

Matsui, T., Sekiguchi, M., Hashimoto, A., Tomita, U., Nishikawa, T. \& Wada, K. (1995). Functional comparison of D-serine and glycine in rodents - the effect on cloned NMDA receptors and the extracellular concentration. J Neurochem 65, 454-458.

Norrander, J., Kempe, T. \& Messing, J. (1983). Construction of 
improved M13 vectors using oligodeoxynucleotide-directed mutagenesis. Gene 26, 101-106.

Osterman, A., Grishin, N. V., Kinch, L. N. \& Phillips, M. A. (1994). Formation of functional cross-species heterodimers of ornithine decarboxylase. Biochemistry 33, 13662-13667.

Park, I. S., Chung-Hung, L. \& Walsh, C. T. (1997). Bacterial resistance to vancomycin: overproduction, purification and characterization of VanC-2 from Enterococcus casseliflavus as a D-Ala:D-Ser ligase. Proc Natl Acad Sci USA 94, 10040-10044.

Reynolds, P. E. (1989). Structure, biochemistry and mechanism of action of glycopeptide antibiotics. Eur J Clin Microbiol Infect Dis 8, 943-950.

Reynolds, P. E., Snaith, H. A., Maguire, A. J., Dutka-Malen, S. \& Courvalin, P. (1994). Analysis of peptidoglycan precursors in vancomycin-resistant Enterococcus gallinarum BM4174. Biochem J 301, 5-8.

Reynolds, P. E., Arias, C. A. \& Courvalin, P. (1999). Gene $\operatorname{van} X Y_{\mathrm{C}}$ encodes both DD-dipeptidase (VanX) and DD-carboxypeptidase (VanY) activities in vancomycin-resistant Enterococcus gallinarum BM4174. Mol Microbiol 36, 341-349.

Sambrook, J., Fritsch, E. F. \& Maniatis T. (1989). Molecular Cloning: a Laboratory Manual, 2nd edn. Cold Spring Harbor, NY: Cold Spring Harbor Laboratory.

Shaw, J. P., Petsko, G. A. \& Ringe, D. (1997). Determination of the structure of alanine racemase from Bacillus stearothermophilus at 1.9-Å resolution. Biochemistry 36, 1329-1342.

Sun, A. Q., Yuksel, K. U. \& Gracet, R. W. (1992). Interactions between the catalytic centers and subunit interface of triosephosphate isomerase probed by refolding, active-site modification, and subunit exchange. J Biol Chem 267, 20168-20174.

Thompson, J. D., Higgins, D. G. \& Gibson, T. J. (1994). CLuSTAL W : improving the sensitivity of progressive multiple sequence alignment through sequence weighting, position-specific gap penalties and weight matrix choice. Nucleic Acids Res 22, 4673-4680.

Truniger, V. \& Boos, W. (1994). Mapping and cloning of gldA, the structural gene of the Escherichia coli glycerol dehydrogenase. $J$ Bacteriol 176, 1796-1800.

Uo, T., Yoshimura, T., Shimizu, S. \& Esaki, N. (1998). Occurrence of pyridoxal 5'-phosphate-dependent serine racemase in silkworm, Bombyx mori. Biochem Biophys Res Commun 246, 31-34.

Walsh, C. T. (1989). Enzymes in the D-alanine branch of bacterial cell wall peptidoglycan assembly. J Biol Chem 264, 2393-2396.

Wasserman, S. A., Walsh, C. T. \& Botstein, D. (1983). Two alanine racemase genes in Salmonella typhimurium that differ in structure and function. J Bacteriol 153, 1439-1450.

Wijsman, H. J. W. (1972). The characterization of an alanine racemase mutant of Escherichia coli. Genet Res 20, 269-277.

Wild, J., Lobocka, M., Walczak, W. \& Klopotowski, T. (1985). Identification of the $d a d X$ gene coding for the predominant isozyme of alanine racemase in Escherichia coli K-12 Mol Gen Genet 198, 315-322.

Wolosker, H., Shieth, K. N., Takahashi, M., Mothet, J., Brady, R. O., Jr, Ferris, C. D. \& Snyder, S. H. (1999a). Purification of serine racemase: biosynthesis of the neuromodulator D-serine. Proc Natl Acad Sci USA 96, 721-725.

Wolosker, H., Blackshaw, S. \& Snyder, S. H. (1999b). Serine racemase: a glial enzyme synthesizing $\mathrm{D}$-serine to regulate glutamate-N-methyl-D-aspartate neurotransmission. Proc Natl Acad Sci USA 96, 13409-13414.

Received 2 December 1999; revised 31 March 2000; accepted 7 April 2000. 